\title{
Quantifying Eating Behavior With a Smart Plate in Patients With Arm Impairment After Stroke
}

\author{
Gert Mertes, Li Ding, Wei Chen, Hans Hallez, Jie Jia and Bart Vanrumste
}

\begin{abstract}
Upper limb motor impairment after stroke has been linked with reduced food intake and affected patients are at risk for malnutrition. The inability to consume food is often proportional to the severity of impairment. Being able to measure the effect of impairment on food intake can lead to additional insights in eating behavior of patients after stroke. In this paper, we propose the use of a smart plate containing weight sensors to extract eating behavior related parameters from individual bites during meals. Data was recorded with the smart plate from 6 patients that had difficulty eating due to impaired arm movement following a stroke. The results indicate that parameters such as bite size and pickup time can be linked to the patient's eating behavior and level of impairment.
\end{abstract}

\section{INTRODUCTION}

Food intake monitoring is the practice of recording ingestive behavior to assist in the diagnosis or management of nutritionrelated disease or conditions, such as malnutrition or obesity [1]. Table embedded scales have been proposed in the literature as a method to automatically measure eating activity [2]-[5]. They typically consist of a weighing surface that measures the consumed food per unit of time during the meal. They can be used to measure beginning and end weights of the meal, indicating the total amount of consumed food. This relatively simple measurement can provide benefits over traditional monitoring methods, which typically rely on pen-and-paper tools requiring manual data entry and may contain mistakes due to human error or deliberate misreporting [1]. Furthermore, these manual methods are time consuming, often resulting in incom-

This research was partially supported by grants from the National Key R\&D Program of China (2017YFE0112000), the Science and Technology Commission of Shanghai Municipality (2017SHZDZX01, 15441901601, 16441905303), KU Leuven (IF/14/038) and Research Foundation Flanders.

G. Mertes is with the Institute of Biomedical Engineering, Department of Engineering Science, University of Oxford, Oxford, U.K. and with the Oxford Suzhou Centre for Advanced Research, Suzhou, Jiangsu, China. The work presented in this paper was done with the Department of Electrical Engineering (ESAT), KU Leuven, Leuven, Belgium. (e-mail: gertmertes@gmail.com)

B. Vanrumste is with the Department of Electrical Engineering (ESAT), KU Leuven, Leuven, Belgium. (e-mail: bart.vanrumste@kuleuven.be)

H. Hallez is with the Department of Computer Science, KU Leuven, Leuven, Belgium. (e-mail: hans.hallez@kuleuven.be).

L. Ding is with the Department of Rehabilitation Medicine, Huashan Hospital, Fudan University, Shanghai, China. (e-mail: leodin@163.com).

W. Chen is with the Center for Intelligent Medical Electronics, Department of Electronic Engineering, Fudan University, Shanghai, China and with Shanghai Key Laboratory of Medical Imaging Computing and Computer Assisted Intervention, Shanghai, China. (e-mail: w_chen@fudan.edu.cn).

J. Jia is with the Department of Rehabilitation Medicine, Huashan Hospital, Fudan University, Shanghai, China and with the National Clinical Research Center for Geriatric Disease, Huashan Hospital Fudan University, Shanghai, China. (e-mail: shannonjj@126.com).

G. Mertes and L. Ding share first-authorship.

Corresponding authors: G. Mertes, J. Jia plete measurements. An automatic measurement system can help alleviate some of these problems and reduce the recording burden on the user. Table embedded scales can furthermore be used to calculated cumulative intake curves. These can be used beyond regular food intake monitoring and can provide researchers or clinicians with more detailed information on the effects of psychological, nutritional or pharmacological changes on human appetite, as well as measure variations in eating rate to detect binge eating [6]-[9].

In previous work, we presented a variation on the table embedded scale in standalone format: a smart plate with integrated weight sensors that can automatically measure food intake [10], [11]. In addition to the total food intake, the system can detect and measure individual bites, which can give a better insight into ingestive behavior by extracting information such as bite size and time spent per bite. These parameters can help to gain insight into eating behavior and provide more contextual information about individual meals or food intake over longer periods of time [12], [13].

Stroke is a medical condition that can drastically change eating behavior. Patients with poor outcomes after stroke are at risk for malnutrition due to reduced appetite and depression [14]. Malnutrition, in turn, has been linked to an increased risk of post-stroke complications [15]. Risk of malnutrition increases with the presence of dysphagia after stroke [16], while impaired arm movement can cause a reduced food intake [17]. Monitoring and nutritional intervention is important in patients with high levels of impairment to prevent malnutrition and increase quality-of-life. To determine a patient's risk of malnutrition and level of impairment after stroke, questionnaires and standardized tests are typically used. While these tests are typically performed regularly during hospital admission, they are no longer taken regularly after release from the hospital. Patients with severe upper limb impairment may be given a personalized dietary plan or adapted cutlery, but their nutritional status is typically not followed up at home.

In this paper, we propose the use of our previously presented smart plate to continuously monitor ingestive behavior of patients after stroke. Patients with impaired arm movement are expected to exert certain behavioral traits during eating, such as reduced eating speed and increased difficulty picking up food. These traits can be detected with the smart plate and can provide clinicians with a better insight into the impairment status of the patient. Furthermore, these parameters can be measured daily and automatically, allowing monitoring at home and giving more insight into the level of impairment and health status of the patient over longer periods of time. 


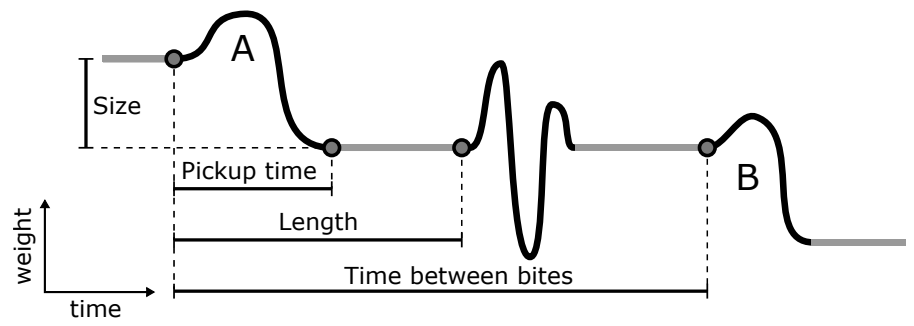

Fig. 1. Bite parameters illustrated on a hand drawn weight signal. The gray parts of the signal are regions of stability.

\section{BITE PARAMETERS}

Figure 2 shows the smart plate system. It consists of a base station with pressure sensors, on top of which an off-the-shelf polymer plate is mounted. It can capture the weight signal of food over time while it is being consumed. Figure 1 shows a hand drawn example of what a weight signal captured with the smart plate generally looks like. There are two bites present in the signal, labeled A and B. Bites typically consist of a short period of instability during which the bite is picked up, followed by a decrease in weight. Following a bite, the signal remains stable as the user consumes the bite and no interaction with the plate takes place. Bites can be detected by looking at the regions of stability surrounding the bite. These regions are marked in the signal in gray. From these regions, certain parameters can be extracted. The region of instability during which a bite is picked up, is the bite pickup time, while the time from when the user begins picking up a bite to the end of the subsequent stable region is the bite length. The region of instability in-between bites A and B can be caused by a number of actions such as re-arranging food or touching the plate, but the signal ultimately stays at the same weight because no food is picked up. During real world unrestricted eating conditions, regions of stability are not guaranteed. The total signal is a more densely packed sequence of periods of stability, periods of instability and decreases in weight. Following a bite, the user may already be manipulating the next bite, shortening or completely eliminating the region of stability. This is one of the challenges of bite detection during unrestricted eating [3].

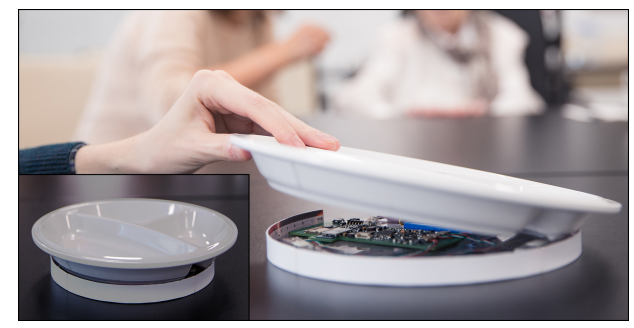

Fig. 2. The smart plate system [10].

\section{DATA}

Data was collected at the Department of Rehabilitation Medicine at Huashan hospital, affiliated to Fudan University in Shanghai, China. Six patients were selected that recently
TABLE I

POST-STROKE PATIENT CHARACTERISTICS.

\begin{tabular}{c|c|c|c} 
ID & age & FMA score $^{\text {a }} \&$ impairment & utensil \\
\hline 1 & 54 & none & chopsticks \\
2 & 84 & 65 (small) & chopsticks \\
3 & 77 & 64 (small) & chopsticks \\
4 & 46 & 27 (severe) & chopsticks \\
5 & 66 & 18 (severe) & spoon \\
6 & 33 & 13 (severe) & spoon \\
\hline
\end{tabular}

a : FMA score of the upper extremities only

suffered a stroke. All patients had an upper limb defect as a result of the stroke, impairing their ability to consume food. Patients were admitted to the rehabilitation ward to regain control over their fine motor skills. To get an overall view of food intake behavior that is independent of external influences or food types, repeat measurements were taken from this group. Over the course of 15 days, patients consumed a meal with the smart plate system every 3 days, resulting in a total of 5 measurements per patient. Food types were kept as consistent as possible, with most meals consisting of rice, meat and vegetables in bite size portions. All patients gave a written informed consent and the study was approved by the Huashan hospital Institutional Review Board.

Table I gives an overview of patient characteristics, ordered by impairment level. The impairment column indicates the level of impairment for the limb that was used to consume the food. The level of impairment was determined with the Fugl-Meyer assessment (FMA) performed by a clinician [18]. The FMA is used to evaluate motor function after stroke. The FMA consists of a standardized set of exercises that are scored based on the physical ability of the patient. Full points (2) are given if the patient can complete the exercise, half points (1) if the exercise can only be completed partially and zero points if the patient is unable to perform the exercise. The complete FMA evaluates motor function of the entire body. For this study, only exercises related to the upper limbs are taken into account. The maximum score obtainable for upper limb exercises is 66. Level of impairment is ranked as follows: $<32$ severe, $32-50$ high, $51-61$ medium, $62-66$ small.

For patient number 1 in the dataset, the defected limb was too severely impaired to consume food, resulting in this patient consuming food with his other, unimpaired, limb, which was also the subject's dominant side. This person can act as a control measurement, since food consumption was more fluent in this patient compared to all other patients. 


\section{METHODS}

The difficulty to consume food due to the upper limb impairment can be seen as a behavioral trait, albeit unwanted. Furthermore, the level of difficulty will change according to the level of impairment. Because the level of impairment is known, we can attempt to link this behavioral trait to the parameters extracted from the smart plate data.

The model we previously presented in [11] is used to detect bites. It uses a Random Forest classifier to detect bites and was trained with data from 18 healthy middle aged to older adults, each consuming a single meal. Bite detection is done based on the assumption that each bite is surrounded by a stable region in the weight signal. A single instance in the classifier consists of a group of three stable regions. True bites were labeled by an observer in the training data. If a true label occurs inbetween the first two stable regions, it is considered a bite. The difference in weight and time between the stable regions are used as features. The trained model is used to detect bites in the post-stroke patient dataset. The model outputs groups of stable regions that are considered a bite. From these regions, the following bite parameters are calculated: bite size, bite length and bite pickup time, as illustrated in Figure 1.

\section{RESUlTS \& DisCuSSION}

Figure 3 shows a box plot containing the distribution of bite sizes per patient. It is difficult to correlate any behavioral parameter to the bite size. There is, however, one observation that can be made. Patients 5 and 6 are the only patients in the dataset that consumed their meals with a spoon, this due to a difficulty with using chopsticks. These patients had the lowest FMA score and the most difficulty eating. The remaining patients all used chopsticks. As shown in the box plot, patients 5 and 6 have a higher median bite size, interquartile range and upper whiskers than the others. This indicates that bite size was larger for these patients, which corresponds to the usage of a spoon. The spoons used were full sized table spoons. More food can be scooped in this kind of spoon than can be picked up with chopsticks. While this is highly related to the food type, the results indicate that the type of utensil may be detected solely from the smart plate data. This can provide further insights into behavioral patterns of smart plate users.

Figure 4 shows the distribution of bite length over all participants. Bite length is defined as the time from when the bite starts to the end of the subsequent stable region in the smart plate data. It is a measure of eating speed, as eating fast will result in the user immediately picking up bite after bite, shortening the time of the subsequent stable region. Eating speed is thus inversely proportionate to bite length. The only three patients in our dataset over the age of 65 , patients 2,3 and 5, exhibit a larger bite length than the other three patients. Furthermore, patient 5 had a severe level of impairment, which may also contribute to the larger bite length. Patients 6 and 4 , however, also had a severe level of impairment and do not exhibit a noticeably large bite length. Bite length may not be a definitive descriptor of impairment, as an impaired patient might still attempt to eat fast.

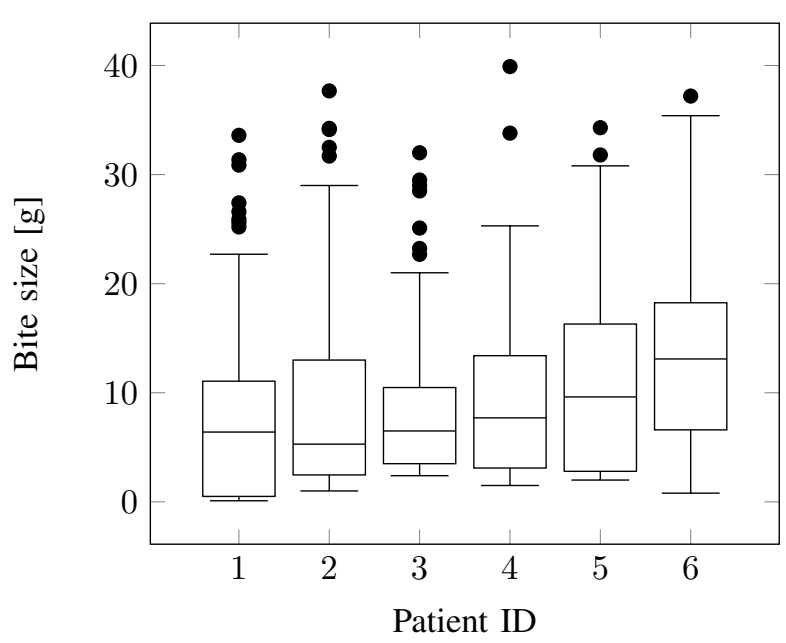

Fig. 3. Box plot showing the distribution of bite size for each patient.

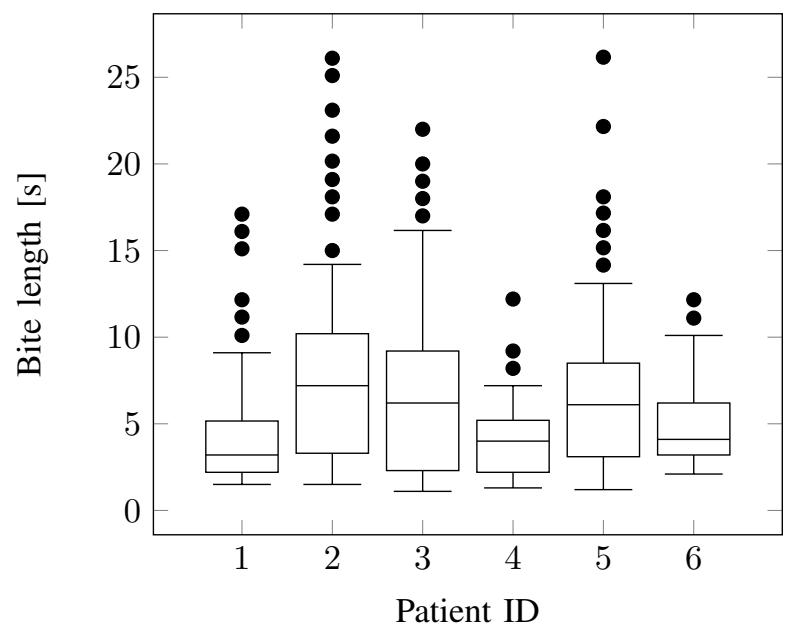

Fig. 4. Box plot showing the distribution of bite length for each patient.

Figure 5 shows the distribution of time required to pick up a bite, also referred to as bite pickup time. Bite pickup time is defined as the time the user first starts interacting with the plate in an attempt to place a bite of food on their utensils, to the time the bite is actually picked up from the plate. For impaired patients, bite pickup time is expected to be long, as the decrease in fine motor skills makes it difficult to pick up food from the plate. They may require multiple attempts to pick up the bite, increasing the bite pickup time. This is also one of the reasons patients 5 and 6 used spoons, which make it easier to pick up food. Patients 4, 5 and 6 with severe impairment show a higher median pickup time than the other patients with lower impairment, high spread and high upper quartile value. This indicates that these patients had the most difficulty piking up bites, which correlates to their impairment status. Patient 2 with small impairment also shows a high interquartile range and high upper whisker, but this is coincidentally also the oldest patient in the dataset, so higher pickup time in this patient may be due to age rather than impairment. The non-impaired patient number 1 shows 


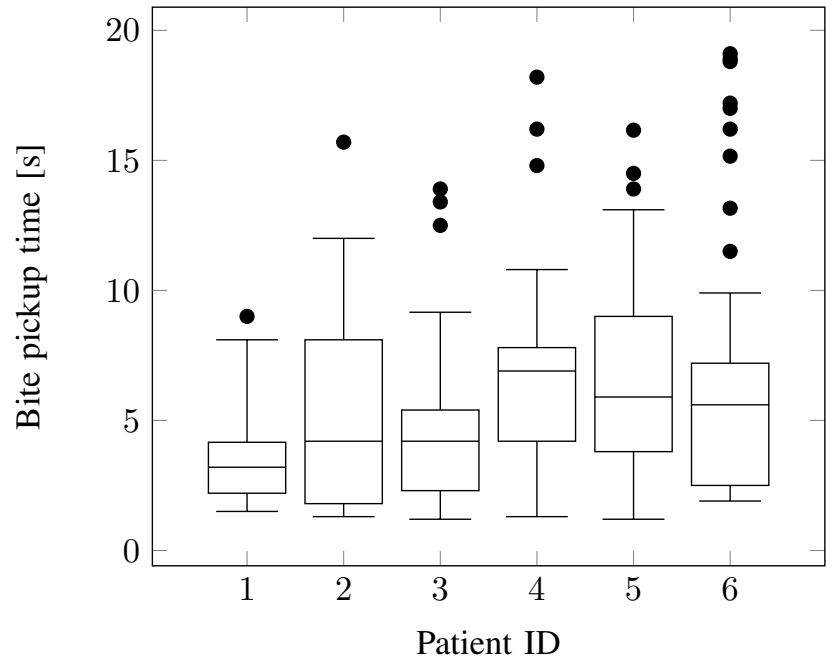

Fig. 5. Box plot with the distribution of the time required to pick up a bite.

the smallest median and spread of all patients with only one outlier and the lowest upper whisker, further adding to the assumption that there may be a correlation between bite pickup time and impairment.

The results show that the strongest indicator of impairment is the bite pickup time, with severely impaired patients having a higher median pickup time. This is in line with the visual observation during meals: these patients had the most difficulty picking up food, while bringing food to the mouth was typically easier. Bite size and length are most likely lesser descriptors of impairment, as an impaired patient may still attempt to take big bites or eat fast. Nevertheless, the results show that there is a measurable difference in the distribution of parameters between patients. This distribution can be linked to the eating behavior of individual subjects.

\section{FUTURE WORK}

While the results of this study are preliminary and exploratory due to the limited size of the dataset, the results show that person-specific parameters can be extracted from the smart plate data. In future work, a larger dataset combined with statistical significance tests can provide more definitive results. Furthermore, extracting these kind of parameters can be useful in different use cases other than follow up after stroke. In the treatment and management of obesity, for example, lifestyle intervention and behavioral therapy play an important role. The ability to link behavioral changes to parameters extracted from the food intake can provide insight into the treatment process as well as better follow up food intake of patients at home.

\section{CONCLUSION}

This paper presented a method of extracting person-specific parameters from individual bites during a meal. The results show that these parameters can be linked to certain behavioral traits, such as difficulty during eating due to impaired arm movement following stroke. While the results are preliminary and further clinical validation is required, we have shown that it is possible to extract these parameters, which can lead the way to future research into a better understanding of eating behavior in patients with different pathologies.

\section{ACKNOWLEDGMENT}

The authors would like to thank the staff and patients of the Hand Function Rehabilitation Department at Shanghai Huashan hospital Jing'An branch for their support and participation in the study.

\section{REFERENCES}

[1] L. Burke, M. Warziski, T. Starrett, J. Choo, E. Music, S. Sereika, S. Stark, and M. Sevick, "Self-monitoring dietary intake: Current and future practices," Journal of Renal Nutrition, pp. 281-290, 2005.

[2] H. R. Kissileff, G. Klingsberg, and T. B. Van Itallie, "Universal eating monitor for continuous recording of solid or liquid consumption in man," American Journal of Physiology-Regulatory, Integrative and Comparative Physiology, vol. 238, no. 1, pp. R14-R22, 1980.

[3] R. S. Mattfeld, E. R. Muth, and A. Hoover, "Measuring the consumption of individual solid and liquid bites using a table embedded scale during unrestricted eating," IEEE journal of biomedical and health informatics, vol. 21, no. 6, pp. 1711-1718, 2017.

[4] K.-h. Chang, S.-y. Liu, H.-h. Chu, J. Y.-j. Hsu, C. Chen, T.-y. Lin, C.-y. Chen, and P. Huang, "The diet-aware dining table: Observing dietary behaviors over a tabletop surface," in International Conference on Pervasive Computing. Springer, 2006, pp. 366-382.

[5] B. Zhou, J. Cheng, M. Sundholm, A. Reiss, W. Huang, O. Amft, and P. Lukowicz, "Smart table surface: A novel approach to pervasive dining monitoring," in Pervasive Computing and Communications (PerCom), 2015 IEEE International Conference on. IEEE, 2015, pp. 155-162.

[6] T. M. Dovey, D. Clark-Carter, E. J. Boyland, and J. C. Halford, "A guide to analysing universal eating monitor data: assessing the impact of different analysis techniques," Physiology \& Behavior, vol. 96, 2009.

[7] H. R. Kissileff, J. Thornton, and E. Becker, "A quadratic equation adequately describes the cumulative food intake curve in man," Appetite, vol. 3, no. 3, pp. 255-272, 1982.

[8] E. M. Bobroff and H. R. Kissileff, "Effects of changes in palatability on food intake and the cumulative food intake curve in man," Appetite, vol. 7, no. 1, pp. 85-96, 1986.

[9] A. Hearty, S. McCarthy, J. Kearney, and M. Gibney, "Relationship between attitudes towards healthy eating and dietary behaviour, lifestyle and demographic factors in a representative sample of irish adults," Appetite, vol. 48, no. 1, pp. 1-11, 2007.

[10] G. Mertes, G. Christiaensen, H. Hallez, S. Verslype, W. Chen, and B. Vanrumste, "Measuring weight and location of individual bites using a sensor augmented smart plate," in 2018 40th Annual International Conference of the IEEE Engineering in Medicine and Biology Society (EMBC). IEEE, 2018, pp. 5558-5561.

[11] G. Mertes, L. Ding, W. Chen, H. Hallez, J. Jia, and B. Vanrumste, "Measuring and localizing individual bites using a sensor augmented plate during unrestricted eating for the aging population," Journal of Biomedical and Health Informatics, in revision.

[12] J. D. Davis, "The microstructure of ingestive behavior," Annals of the New York Academy of Sciences, vol. 575, no. 1, pp. 106-121, 1989.

[13] H. Kissileff and J. Guss, "Microstructure of eating behavior in humans." Appetite, vol. 36, no. 1, p. 70, 2001.

[14] L. Perry and S. Mclaren, "An exploration of nutrition and eating disabilities in relation to quality of life at 6 months post-stroke," Health \& social care in the community, vol. 12, no. 4, pp. 288-297, 2004.

[15] S.-H. Yoo, J. S. Kim, S. U. Kwon, S.-C. Yun, J.-Y. Koh, and D.-W. Kang, "Undernutrition as a predictor of poor clinical outcomes in acute ischemic stroke patients," Archives of neurology, vol. 65, 2008.

[16] N. C. Foley, R. E. Martin, K. L. Salter, and R. W. Teasell, "A review of the relationship between dysphagia and malnutrition following stroke," Journal of Rehabilitation Medicine, vol. 41, no. 9, pp. 707-713, 2009.

[17] S. McLaren and J. Dickerson, "Measurement of eating disability in an acute stroke population," Clinical Effectiveness in Nursing, vol. 4, 2000.

[18] A. R. Fugl-Meyer, "Post-stroke hemiplegia assessment of physical properties," Scand J Rehabil Med Suppl, vol. 7, pp. 85-93, 1980. 